\title{
Extended field or pelvic intensity-modulated radiotherapy with concurrent cisplatin chemotherapy for the treatment of post-surgery multiple pelvic lymph node metastases in cervical cancer patients: a randomized, multi-center phase II clinical trial
}

\author{
Weiming Luo ${ }^{1,2,3}$, Yunhai $\mathrm{Li}^{3}$, Guihao $\mathrm{Ke}^{1,2}$, Xiaohua $\mathrm{Wu}^{1,2}$, Xiao Huang ${ }^{1,2}$ \\ ${ }^{1}$ Department of Gynaecology, Fudan University, Shanghai Cancer Center, Shanghai, China; ${ }^{2}$ Department of Oncology, Shanghai Medical College, \\ Fudan University, Shanghai, China; ${ }^{3}$ Department of Radiation Oncology, Fudan University, Shanghai Cancer Center Minhang Branch, Shanghai, \\ China \\ Contributions: (I) Conception and design: W Luo, X Huang; (II) Administrative support: W Luo, X Huang; (III) Provision of study materials or \\ patients: Y Li, G Ke, X Wu; (IV) Collection and assembly of data: W Luo, Y Li, G Ke, X Wu; (V) Data analysis and interpretation: W Luo, Y Li; (VI) \\ Manuscript writing: All authors; (VII) Final approval of manuscript: All authors. \\ Correspondence to: Xiao Huang. Department of Gynaecology, Fudan University, Shanghai Cancer Center, 270 Dong'An Road, Shanghai 200032, \\ China; Department of Oncology, Shanghai Medical College, Fudan University, Shanghai, China. Email: smilehuangxiao@163.com.
}

Background: To prospectively compare the outcomes and side effects between groups of postoperative cervical cancer patients with multiple pelvic lymph node metastases who were treated with extended field or pelvic intensity-modulated radiotherapy (IMRT) with concurrent cisplatin chemotherapy.

Methods: Cervical carcinoma patients with International Federation of Gynecology and Obstetrics (FIGO) stage Ib-IIa, who underwent radical hysterectomy and had histologically confirmed multiple $(\geq 2)$ pelvic lymph node metastases, were enrolled into this study. The patients were randomly assigned to pelvicIMRT or extended field-IMRT (45 Gy/25 Fx) group. Patients in either group received concurrent cisplatin chemotherapy $(40 \mathrm{mg} / \mathrm{m} 2)$ starting on the first day of irradiation.

Results: Until December 31th 2017, 129 patients were initially enrolled into this study. During the study, 3 patients were dropped out due to either incompletion of the study or exclusion by the criteria. Consequently, 64 patients completed pelvic-IMRT, and 62 patients completed extended field-IMRT. Median follow-up period was 61.30 months in the extended field-IMRT group and 60.60 months in the pelvic-IMRT group. Five-year actuarial survival probability was 0.759 (95\% CI: $0.619-0.854)$ in the extended field-IMRT group which was not significantly different from that of the pelvic-IMRT group [0.824 (95\% CI: 0.690-0.905), $\mathrm{P}=0.442$ ]. Similarly, the five-year progression-free probability was 0.720 (95\% CI: $0.576-0.822$ ) in the extended field-IMRT group, which was not significantly different from that of the pelvic-IMRT group [0.781 (95\% CI: 0.637-0.874), $\mathrm{P}=0.389$ ]. In addition, there was no significant difference between the two groups in hematology and gastrointestinal tract toxicities.

Conclusions: Post-operative pelvic-IMRT or extended field-IMRT with concurrent cisplatin chemotherapy had similar outcomes in terms of survival rates and adverse events in cervical carcinoma patients at FIGO stage Ib-IIa with multiple pelvic lymph nodes metastases.

Keywords: Cervical carcinoma; intensity-modulated radiotherapy (IMRT); extended field, para-aortic lymph node; side effects

Submitted Jul 22, 2020. Accepted for publication Nov 06, 2020.

doi: $10.21037 /$ tcr-20-2573

View this article at: http://dx.doi.org/10.21037/tcr-20-2573 


\section{Introduction}

Cervical cancer is the $2^{\text {nd }}$ most common malignancy for women in the world. For the patients with Ib-IIa stage cervical cancer, radical hysterectomy followed by radiotherapy is the main therapeutic method, and the 5 -year survival rate of cervical cancer is $80-90 \%(1,2)$. Postoperative recurrence of cervical cancer largely depends on the following risk factors: pelvic lymph node metastasis, positive surgical margins, parametrial invasion, large tumor size, vascular invasion, and deep cervical stromal invasion. Of the aforementioned risk factors, lymph node metastasis is the most important predictor for patients' survival. In this regard, studies indicated that prognosis of cervical cancer patients was worse if the patient had para-aortic (mainly abdominal aorta) lymph node metastasis (3-5). The number of the pelvic lymph node with cervical cancer cell metastasis could serve as a predictor of para-abdominal aorta lymph node metastasis: incidence of para-abdominal aorta lymph node metastasis was $0.5 \%$ if the number of pelvic lymph node metastasis was $\leq 1$, while it was $27.6 \%$ if the number was $\geq 2$ (6).

Therefore, cervical cancer patients with the aforementioned risk factors are often given radiotherapy and concurrent chemotherapy after surgical resection (5,7-11). In this context, in order to improve cervical cancer patient's survival rate, the Radiation Therapy Oncology Group (RTOG) had conducted a study on the extended field of pelvic radiotherapy for cervical cancer patients (12). While post-operative expanded pelvic filed radiotherapy could significantly improve survival rate in the stage Ib-IIa cervical cancer patients $(13,14)$, the efficacy of the extended pelvic field radiotherapy on the overall survival for the patients with early-stage cervical cancer is still controversial and remains to be investigated (15). In addition, modern trials such as RTOG1203 used IMRT for post-operative pelvic radiation but did not extend to cover para-aortic fields, and thus, toxicity of extend filed radiation therapy (EFRT) with IMRT is not known. The current study was, therefore, designed to explore and compare the efficacy as well as the incidence of adverse events in cervical cancer patients who randomly received pelvic-IMRT or extended pelvic field (abdominopelvic)-IMRT plus concurrent chemotherapy after radical hysterectomy and pelvic only lymph node dissection.

We present the following article in accordance with the CONSORT reporting checklist (available at http://dx.doi. org/10.21037/tcr-20-2573).

\section{Methods}

\section{Patient enrollment and randomization}

Inclusion criteria: (I) age: 18-70 years old; ECOG score: 0-2; (II) cervical cancer patients at stage Ib-IIa by International Federation of Gynecology and Obstetrics (FIGO) [2009] staging criteria. Patients had not received any adjuvant therapy before radical hysterectomy plus pelvic lymphadenectomy with or without abdominal paraaortic lymphadenectomy. Intraoperative exploration (instant biopsy and pathological examination when paraaortic lymph node seemed enlarged) indicated no signs of abdominal para-aortic lymph nodes metastasis or lymph node biopsy revealed negative for metastasis; (III) at least 2 or more pelvic lymph node metastasis; (IV) postsurgery histology confirmed as squamous carcinoma, adenocarcinoma, adenosquamous cancer, or adenoid basal cell cancer; (V) postoperative hemoglobin $\geq 10 \mathrm{~g} / \mathrm{dL}$, white blood cell $\geq 4.0 \times 10^{9} / \mathrm{L}$, neutrophil $\geq 2.1$, platelet $\geq 80 \times 10^{9} / \mathrm{L}$, blood creatinine $\leq 2.0 \mathrm{mg} / \mathrm{dL}$, aspartate aminotransferase $\leq 2 \mathrm{U} / \mathrm{L}$; (VI) pre- and post-surgery pelvic and abdominal CT or MRI scanning images, and pre-surgery chest plain radiograph or CT scanning images were available; (VII) patients who signed the consent form.

Exclusion criteria: patients with confirmed metastasis of abdominal para-aortic lymph node or common iliac lymph node; confirmed involvement of vaginal stump; confirmed involvement of para-uterus tissues; confirmed distant metastasis; angina pectoris, heart dysfunction, myocardial infarction, acute infection, or liver and kidney dysfunction; allergy to chemotherapeutic reagents; history of abdominal or pelvic radiotherapy; failure of follow-up.

The study was conducted in accordance with the Declaration of Helsinki (as revised in 2013). Study protocol of the current study was approved by IRB committee of the Fudan University. The clinical registration number of this study is ChiCTR-IPR-14005499. Patients were randomly grouped by the number drawn from a sealed envelope. All patients gave their written informed consent.

\section{Radiotherapy and concurrent chemotherapy}

Intensity-modulated radiotherapy (IMRT) was used for both extended field (abdominopelvic) radiation and pelvic radiation. Patients were at a supine position and fixed with $B$ pillow foot pads/abdominal board. Patients were allowed to drink $800 \mathrm{~mL}$ water $30 \mathrm{~min}$ prior to simulated positioning from T10 to $5 \mathrm{~cm}$ below ischial tuberosity by CT scan 
with $5 \mathrm{~cm}$ fraction thickness. Patients drank an additional $800 \mathrm{~mL}$ water prior to the radiotherapy in order to fill the bladder. The CT images determined clinical target volume (CTV) following CBCT guidelines.

For the extended field-IMRT group, CTV included paracolpium, at least $3 \mathrm{~cm}$ of postoperative vaginal stump, obturator lymph nodes, internal iliac lymph nodes, external iliac lymph nodes, common iliac lymph nodes, anterior sacral lymph nodes, and para-abdominal aorta lymph nodes. Specifically, (I) common iliac lymph nodes: expanded by $7 \mathrm{~mm}$ around the artery; rear and side edge expanded to the vertebra and psoas muscle. (II) External iliac lymph nodes: included 3 groups, that is, external group, internal group, and anterior group. Extended by $7 \mathrm{~mm}$ for the internal and anterior groups, and by $17 \mathrm{~mm}$ for the external group along with the iliopsoas based on the previous study (16). (III) Obturator lymph nodes: extended towards and along the pelvic wall by $18 \mathrm{~mm}$, which connected to the CTV of the internal and external iliac artery. (IV) Internal iliac lymph nodes: extended around the artery by $7 \mathrm{~mm}$ to the pelvic wall. (V) Anterior sacral lymph nodes: extended by $10 \mathrm{~mm}$ into the pelvis and connected to the CTV of common iliac lymph nodes. (VI) Para-abdominal aorta lymph nodes: expanded by $2 \mathrm{~cm}$ towards left, by $0.5 \mathrm{~cm}$ to front, and by $1 \mathrm{~cm}$ right of the inferior vena cava; up to the top edge of number 1 lumbar, bottom to the line of CTV for common iliac lymph nodes.

For the pelvic-IMRT group, CTV included paracolpium, at least $3 \mathrm{~cm}$ of the postoperative vaginal stump, obturator lymph nodes, anterior sacral lymph nodes, and internal iliac lymph nodes, external iliac lymph nodes, and common iliac lymph nodes. Planning target volume (PTV) was defined as extending by $8 \mathrm{~mm}$ from the CTV. Radiation dose of 45 Gy/25 Fx was delivered by Pinnacle treatment planning system (TPS) with requirement of V97\% PTV $>45$ Gy, V110\% PTV (45 Gy) <20\%, V93\% PTV (45 Gy) $<1 \%$. Radiation limitation to the organs was as following: intestine V40 $<30 \%$. rectum V50 $<35 \%$, bladder V 50 $<35 \%$, femoral head V $30<20 \%$. Kidney V15 $<50 \%$.

Concurrent chemotherapy was initiated on day 1 of radiotherapy with cisplatin $40 \mathrm{mg} / \mathrm{m}^{2}$ intravenously, once per week, 5 weeks (17).

\section{Outcome assessment}

During the treatment, routine blood test was performed once a week; tests for liver function, kidney function, and electrolytes were carried out every two weeks. During the follow-up period, the patients were examined at one month after completion of the radiotherapy; every 3 months within 2 years after completion of the therapy; every 6 months from 2 to 5 years after completion of the therapy; and once a year after 5 years of the completion of radiotherapy. At every follow-up visit, examinations included routine tests of blood, urine, and stool; liver and kidney functions; electrolytes; tumor biomarkers; chest CT scan; enhanced abdominal CT scan; and enhanced pelvic MR. Efficacy of the treatment was evaluated by Response Evaluation Criteria in Solid Tumors (RECIST) version 1.1, and adverse events were assessed by Common Terminology Criteria for Adverse Events (CTCAE) version 3.0 (18). Adverse events were assessed weekly during the treatment and at one month, 3 months, and 6 months after completion of the treatment.

\section{Statistical analysis}

Discrete variables were expressed by frequency (\%), and Chi-square test or Fisher exact probability test was used. Kaplan-Meier curve was used for survival analysis and logrank was used to compare the survival rates between the groups. Multi-variance cox regression analysis was used for mortality and disease progression analysis. Overall survival was defined as from the date of enrolling into this study to the date of death. Disease progression timeline was defined as from the first date of radiotherapy to date of disease progression (getting worse) was observed or death of the patient. SAS 9.3 was used for all analyses. $\mathrm{P}$ value $<0.05$ was considered as significant.

\section{Results}

\section{General characteristics of the patients}

A total of 129 cervical cancer patients, who received radical hysterectomy followed by radiotherapy and concurrent chemotherapy with cisplatin from January $1^{\text {st }}, 2012$ to December $31^{\text {st }}$, 2017, were initially enrolled in this study. Of them, 2 patients were at stage III by FIGO and one patient did not complete the treatment, and thus, 126 cases were enrolled in the final analysis. Of the 126 patients, 62 patients received extended field-IMRT and 64 patients received pelvic-IMRT. Number of the patients who had at least 4 lymph nodes metastasis was significantly higher in the group treated with extended field-IMRT (19 out of 62, $30.65 \%$ ) compared to that of the pelvic-IMRT group (9 out 
of $64,14.6 \%, \mathrm{P}=0.025$, Table 1$)$. There was no significant difference between the two groups in other parameters including age, tumor size, and FIGO stage (Table 1).

\section{Efficacy of the treatment}

Majority of the patients in either extended field-IMRT (45 out of $62,72.5 \%$ ) or pelvic-IMRT group (52 out of 64 , $81.3 \%$ ) completed therapies. Time gap between radical hysterectomy and radiotherapy initiation was 45.50 days in the extended field-IMRT group and 44.00 days in the pelvic-IMRT group (Table 2). The total dose of radiation was identical (4,500 Gy) in the two groups (Table 2). Median follow-up period was 61.30 months in the extended fieldIMRT group and 60.60 months in the pelvic-IMRT group (Table 2).

Thirteen out of 62 patients died in the extended fieldIMRT group, while 9 out of 64 patients died in the pelvicIMRT group during the follow-up period. The survival probability in the two groups was analyzed by Log-Rank test. As shown in Figure 1, one-year, three-year, and fiveyear survival probability was 0.983 (95\% CI: 0.884-0.998), 0.824 (95\% CI: $0.698-0.901$ ), and 0.759 (95\% CI: $0.619-$ $0.854)$, respectively, in the extended field-IMRT group, and 0.981 (95\% CI: 0.874-0.997), 0.845 (95\% CI: 0.714-0.919), and 0.824 (95\% CI: $0.690-0.905)$, respectively, in the pelvic-IMRT group. There was no significant difference between the two groups $(\mathrm{P}=0.442)$.

Seventeen out of 62 patients had progressive cancer recurrence in the extended field-IMRT group, while 12 out of 64 patients had progress in the pelvic-IMRT group during the follow-up period. Progression-free probability in the two groups was also analyzed by Log-Rank test and presented in Figure 2. One-year, three-year, and fiveyear progression-free probability was 0.878 (95\% CI: 0.762-0.940), 0.789 (95\% CI: 0.658-0.874), and 0.720 (95\% CI: 0.576-0.822), respectively, in the extended fieldIMRT group, and 0.869 (95\% CI: 0.746-0.936), 0.812 (95\% CI: 0.678-0.894), and 0.781 (95\% CI: 0.637-0.874), respectively, in the pelvic-IMRT group. There was no significant difference between the two groups $(\mathrm{P}=0.389)$.

Analysis on mortality and disease progression revealed that tumor size (maximum diameter of the tumor $\geq 5$ or $<5)$ was significantly associated with mortality and disease progression [Model 1: HR (95\% CI): 3.509 (1.353-9.100), $\mathrm{P}=0.010$ for mortality and 2.664 (1.202-5.904), $\mathrm{P}=0.0158$ for disease progression; Model 2: HR (95\% CI): 4.295 (1.715-10.753), $\mathrm{P}=0.002$ for mortality and 3.041 (1.413-
6.547), $\mathrm{P}=0.0045$ for disease progression, respectively, Table 3].

\section{Adverse effect}

None of the participants had adverse event worse than grade V. As shown in Table 4, while hematological toxicity was the major side effects, there was no significant difference between the two groups in hematological toxicity, gastrointestinal reaction, liver toxicity, kidney toxicity or cardiovascular toxicity $(\mathrm{P}>0.05)$.

\section{Discussion}

In this prospective study, cervical cancer patients, who had a radical hysterectomy, were randomized to receive either pelvic-IMRT or extended field-IMRT concurrent with chemotherapy. It was found that neither the overall survival probability nor the progression-free probability was significantly different between the two groups. In addition, there was no significant difference between the two groups in hematological toxicity, gastrointestinal reaction, liver toxicity, kidney toxicity or cardiovascular toxicity. These findings suggested that the efficacy of extended field (abdominopelvic) radiotherapy is similar to that of pelvic radiotherapy; that post-operative radiation on the extended field did not increase the adverse events of hematology and gastrointestinal tract in the cervical cancer patients with pelvic lymph node metastasis.

The lymphatic system is the major route for cervical cancer metastasis, which often occurs in a stepwise progression. In this regard, cervical cancer patients with positive pelvic lymph node metastasis often have metastasis to the para-aortic lymph nodes, especially paraabdominal aorta lymph nodes, in approximately $10-25 \%$ patients (19). Para-aortic lymph node metastasis often indicates poor prognosis for cervical cancer patients $(3,20,21)$. Studies indicated that lymph node metastasis is the most important predictor for cervical cancer patients $(20,22,23)$. Findings of clinical surgery indicated that paraaortic lymph node involvement was up to $29 \%$ of the cervical cancer patients (24) and even more common in patients with pelvic lymph node metastases (6). Cervical cancer cells often invade into the lymph nodes located in the lower part of the pelvic cavity and migrate to the lymph nodes of the upper part of pelvic cavity including common iliac lymph nodes, and then further to the paraabdominal aorta lymph nodes. Risk of para-abdominal 
Table 1 Characteristics before the treatment

\begin{tabular}{|c|c|c|c|c|c|}
\hline Parameters & Extended field ( $\mathrm{N}=62)$ & Pelvic $(\mathrm{N}=64)$ & Test methods & Statistics & $P$ \\
\hline Group by age (\%) & & & & 0.172 & 0.678 \\
\hline$\geq 45$ & $40(64.52)$ & $39(60.94)$ & Chi-square & & \\
\hline$<45$ & $22(35.48)$ & $25(39.06)$ & & & \\
\hline Group by diameter (\%) & & & & 0.018 & 0.893 \\
\hline$\geq 5$ & $23(37.10)$ & $23(35.94)$ & Chi-square & & \\
\hline$<5$ & $39(62.90)$ & $41(64.06)$ & & & \\
\hline$\geq 4$ & $19(30.65)$ & $9(14.06)$ & Chi-square & & \\
\hline $2-3$ & $43(69.35)$ & $55(85.94)$ & & & \\
\hline FIGO stage (\%) & & & Fisher's Exact Test & - & 0.319 \\
\hline IB & $22(35.48)$ & $29(45.31)$ & & & \\
\hline IIA & $39(62.90)$ & $35(54.69)$ & & & \\
\hline IIB & $1(1.61)$ & $0(0.00)$ & & & \\
\hline Histological classification (\%) & & & Fisher's Exact Test & - & 0.441 \\
\hline Vaginal vault metastasis (\%) & & & & 0.127 & 0.722 \\
\hline No & $30(48.39)$ & $33(51.56)$ & Chi-square & & \\
\hline Yes & $32(51.61)$ & $31(48.44)$ & & & \\
\hline Vaginal metastasis (\%) & & & & 0.670 & 0.413 \\
\hline No & $57(91.94)$ & $56(87.50)$ & Chi-square & & \\
\hline Yes & $5(8.06)$ & $8(12.50)$ & & & \\
\hline Depth of cervix invasion (\%) & & & & 1.466 & 0.226 \\
\hline$>$ Deep 1/3 & $38(62.30)$ & $33(51.56)$ & Chi-square & & \\
\hline Middle or deep $1 / 3$ & $23(37.70)$ & $31(48.44)$ & & & \\
\hline Vascular invasion (\%)* & & & & 0.012 & 0.913 \\
\hline No & $15(25.86)$ & $16(25.00)$ & Chi-square & & \\
\hline Yes & $43(74.14)$ & $48(75.00)$ & & & \\
\hline
\end{tabular}

*, one case or 4 cases were dropped out. FIGO, International Federation of Gynecology and Obstetrics. 
Table 2 Comparison of radiotherapy and follow-up period

\begin{tabular}{|c|c|c|c|c|c|}
\hline Parameters & Extended field ( $\mathrm{N}=62)$ & Pelvic $(\mathrm{N}=64)$ & Test methods & Statistics & $\mathrm{P}$ \\
\hline $\begin{array}{l}\text { Total dose of radiotherapy (Gy), } \\
\text { median (IQR) }\end{array}$ & $4,500.00(4,500.00,4,500.00)$ & $4,500.00(4,500.00,4,500.00)$ & $\begin{array}{l}\text { Wilcoxon two } \\
\text { sample test }\end{array}$ & 0.110 & 0.913 \\
\hline $\begin{array}{l}\text { Median follow-up period (m), } \\
\text { median (IQR) }\end{array}$ & $61.30(35.90,76.80)$ & $60.60(23.25,79.15)$ & $\begin{array}{l}\text { Wilcoxon two } \\
\text { sample test }\end{array}$ & -0.032 & 0.975 \\
\hline
\end{tabular}

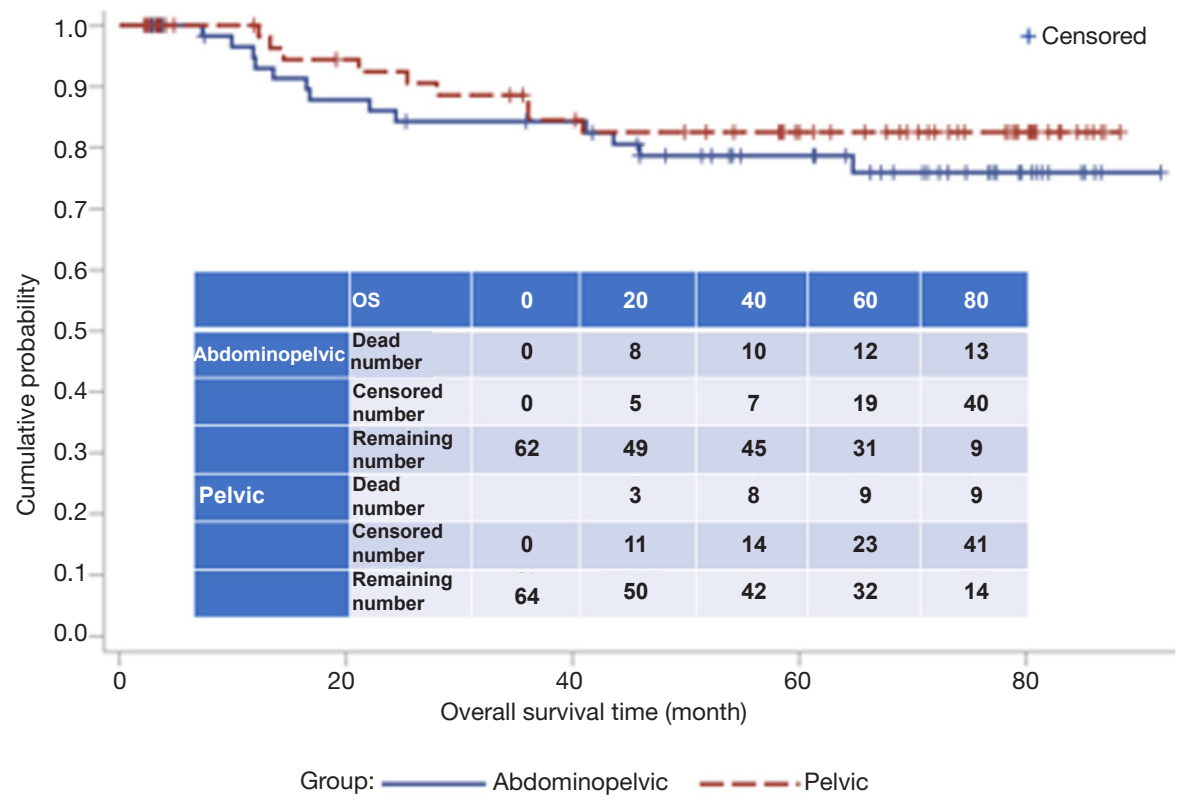

Figure 1 Comparison of overall survival.

aorta lymph node metastasis is highly associated with the number of pelvic lymph node involvement (25). In this regard, Zhou reported that $40-70 \%$ of 334 cervical cancer patients had para-abdominal aorta metastasis if the patients had 2 or more pelvic lymph nodes metastases (26). Studies by the Gynecology Oncology Group (GOG) revealed that metastasis to the paraabdominal aorta lymph nodes was positively correlated with cervical cancer stages, that is, stage I, II, and III had $5 \%, 17 \%$, and $25 \%$ of para-abdominal aorta lymph node metastasis, respectively (27). Therefore, prophylactic extended-filed (abdominopelvic) radiotherapy has been suggested to treat those with locally advanced cervical cancer in order to sterilize micrometastasis and mitigate the risk of distant relapse.

Postoperative concurrent radiotherapy and chemotherapy is a conventional therapeutic strategy for cervical cancer patients who potentially have para-aortic lymph node metastasis $(10,11,28,29)$. In order to improve the survival of cervical cancer patients with para-aorta lymph node metastasis, RTOG conducted a phase III clinical trial in 1995. It was found that expanded pelvic filed radiotherapy could significantly improve 10-year survival rate in the stage Ib-IIa cervical cancer patients (12). Another clinical trial by RTOG in 1999 compared radical pelvic radiotherapy plus concurrent chemotherapy with cisplatin and fluorouracil versus extended field radiotherapy plus concurrent chemotherapy with the aforementioned reagents. It was found that the survival rate of radical pelvic radiotherapy plus concurrent chemotherapy was superior to that of the radically extended field pelvic radiotherapy plus concurrent chemotherapy in patients with Ib-IIa cervical cancer (30). In a retrospective study, Zhang et al. compared the efficacy of expanded field radiotherapy plus 


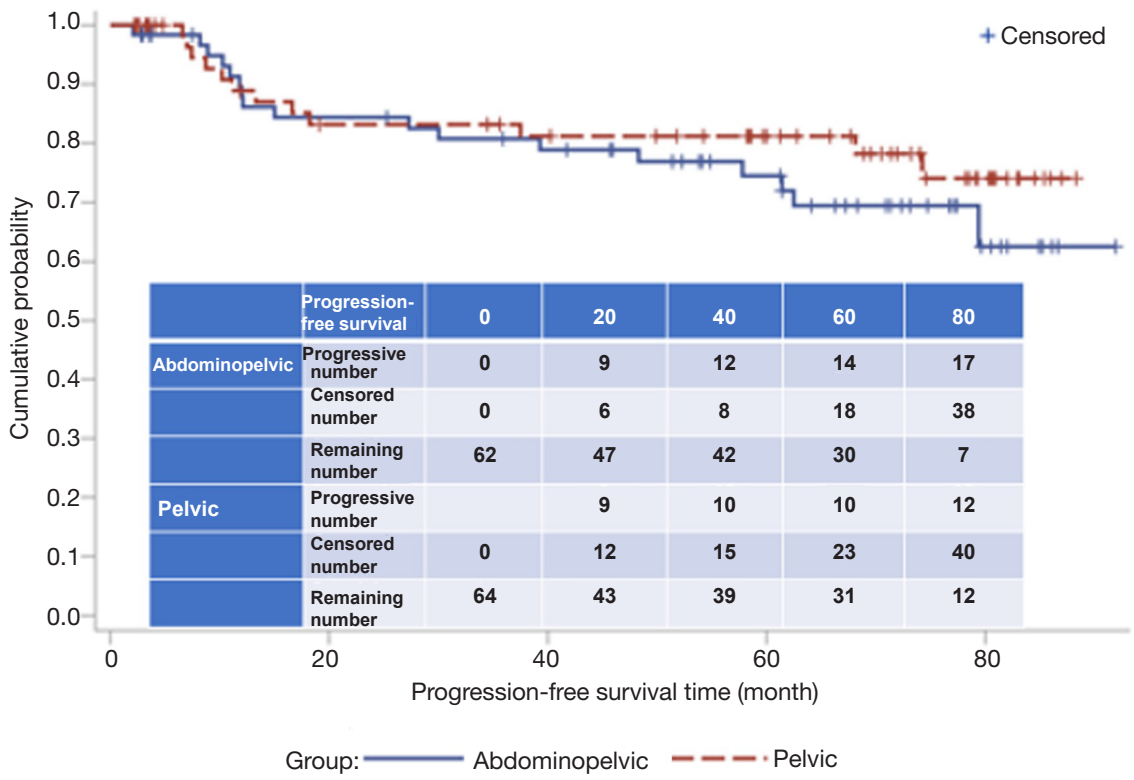

Figure 2 Comparison of progress-free survival.

Table 3 Multi-variance COX regression analysis on mortality and progression

\begin{tabular}{|c|c|c|c|c|c|c|}
\hline \multirow{2}{*}{ COX regression } & \multirow{2}{*}{$\begin{array}{l}\text { Independent } \\
\text { variables }\end{array}$} & \multirow{2}{*}{ Classification level } & \multicolumn{2}{|c|}{ Risk of death } & \multicolumn{2}{|c|}{ Risk of progression } \\
\hline & & & $\mathrm{HR}(95 \% \mathrm{Cl})$ & $\mathrm{P}$ & $\mathrm{HR}(95 \% \mathrm{Cl})$ & $P$ \\
\hline \multirow[t]{5}{*}{ Model 1} & Radiotherapy & Ab- $p^{*}$ vs. pelvic & $1.053(0.427-2.595)$ & 0.910 & $1.157(0.534-2.508)$ & 0.7121 \\
\hline & Age (y) & $\geq 45$ vs. $<45$ & $1.355(0.514-3.574)$ & 0.539 & $0.984(0.448-2.161)$ & 0.9674 \\
\hline & Histology & Squamous vs. others & $0.67(0.203-2.216)$ & 0.512 & $0.690(0.225-2.116)$ & 0.5162 \\
\hline & Max diameter & $\geq 5$ vs. $<5$ & $3.509(1.353-9.100)$ & 0.010 & $2.664(1.202-5.904)$ & 0.0158 \\
\hline & $\mathrm{N}$ of $\mathrm{LN}-\mathrm{PM}^{\#}$ & $\geq 4$ vs. $<4$ & $1.542(0.506-4.696)$ & 0.446 & $1.433(0.561-3.661)$ & 0.4526 \\
\hline \multirow[t]{2}{*}{ Model 2} & Radiotherapy & Ab-p* vs. pelvic & $1.171(0.483-2.835)$ & 0.727 & $1.213(0.563-2.617)$ & 0.6218 \\
\hline & Max diameter & $\geq 5$ vs. $<5$ & 4.295 (1.715-10.753) & 0.002 & 3.041 (1.413-6.547) & 0.0045 \\
\hline
\end{tabular}

*, Ab-p: abdominopelvic; ", number of lymph nodes in pelvic with metastasis.

chemotherapy, expanded field radiotherapy only, pelvic radiotherapy plus chemotherapy, and pelvic radiotherapy only for cervical cancer $>4 \mathrm{~cm}$ and pelvic lymph node metastasis. They found that extended field radiotherapy alone was superior to pelvic radiotherapy alone, however, there was no difference when concurrent chemotherapy was added to the radiotherapy (31). Recently, Oh et al. (5) reported that prophylactic radiotherapy for para-aortic lymph node metastases did not have an additional benefit in patients with pelvic lymph node-positive cervical cancer treated with concurrent chemoradiotherapy (CCRT). Similarly, the current study demonstrated that there was no significant difference in terms of efficacy and adverse events between the pelvic-IMRT plus concurrent chemotherapy and extended field-IMRT plus concurrent chemotherapy. These findings suggested that post-operative extended field-IMRT plus concurrent chemotherapy is not superior to pelvicIMRT plus concurrent chemotherapy for the treatment of cervical cancer with pelvic lymph node metastasis.

In a previously reported retrospective study on 25 
Table 4 Comparison of adverse events

\begin{tabular}{|c|c|c|c|c|c|}
\hline Grade of adverse events & Extended field $(\mathrm{N}=62)$ & Pelvic $(\mathrm{N}=64)$ & Test methods & Statistics & $P$ \\
\hline 0 & $10(16.13)$ & $13(20.31)$ & & & \\
\hline 1 & $8(12.90)$ & $9(14.06)$ & & & \\
\hline 2 & $22(35.48)$ & $26(40.63)$ & & & \\
\hline 4 & $2(3.23)$ & $2(3.13)$ & & & \\
\hline Gastrointestinal reaction (\%) & & & Chi-square & 0.309 & 0.857 \\
\hline 0 & $44(70.97)$ & $48(75.00)$ & & & \\
\hline 1 & $13(20.97)$ & $11(17.19)$ & & & \\
\hline 0 & $61(98.39)$ & 59 (92.19) & & & \\
\hline 1 & $1(1.61)$ & $4(6.25)$ & & & \\
\hline 2 & $0(0.00)$ & $1(1.56)$ & & & \\
\hline
\end{tabular}

cervical cancer patients (FIGO stage: Ib-IIb, and 2 or more pelvic lymph nodes metastases confirmed by post-operative histology examination), we found that 3-year PFS and OS were $63 \%$ and $76 \%$, respectively, when they were treated with extended field radiotherapy plus chemotherapy (32). However, $76 \%$ of these patients had 1-2 grade decrease in leukocytes and $16 \%$ had 3-4 grade decrease in leukocytes; $60 \%$ had 1-2 grade gastrointestinal reaction and $4 \%$ had 3-4 grade gastrointestinal reaction (32). Similarly, Chen et al. reported $4.5 \%$ of the patients, who had extendedfield radiotherapy plus concurrent chemotherapy, had late phase adverse events (33). Based on the findings of the previous study, the current prospective study was designed to further explore whether extended field (abdominopelvic) radiotherapy plus concurrent chemotherapy is superior to pelvic radiotherapy plus chemotherapy for the cervical cancer patients who had 2 or more pelvic lymph node metastases. To accomplish this, in the current study, patients with common iliac lymph node metastasis were excluded for that common iliac lymph node metastasis is considered as a dependent risk factor of early invasion of cervical cancer cells into the para-abdominal aortic lymph nodes (34). In addition, compared to the previous retrospective study (32), this study was modified with advanced radiotherapy techniques in the following aspects: dose and three-dimensional distribution of radiation in the important organs were more precise and thus protected the normal tissues of the organs; simultaneous radiotherapy on multiple organs and multiple fields, which resulted in optimization and improvement of the therapeutic radiation dose and efficacy. With these modifications of radiotherapy, hematological and gastrointestinal adverse events in the patients with extended field-IMRT plus concurrent chemotherapy were not significantly increased compared to those in patients with pelvic-IMRT plus chemotherapy, and there were no significant differences in terms of 5 -year survival probability and 5 -year progression-free probability between the two groups. The following factors might be associated with the lack of advantage in the efficacy of extended-field radiotherapy. (I) Studies indicated that the size and total number of metastatic lymph nodes is associated with patients' prognosis $(29,35)$. In the current study, while the size of the metastatic lymph nodes was not significantly different between the two groups, the number of positive pelvic lymph node metastasis $(\geq 4)$ was significantly higher in the extended field-IMRT group than that in the pelvic-IMRT group. (II) Extended field radiotherapy could more significantly suppress patients' immunity, which is a crucial factor affecting outcomes of tumor treatment. (III) While this was a prospective, randomized and multicenter study, the number of cases was limited and the follow-up period was short. 
Recently, several studies reported reduction of adverse effects of radiotherapy or CCRT through strategies such as a sequential strategy of systemic chemotherapy followed by radiotherapy, and IMRT (36,37). Particularly, the high precision technique of IMRT could spare adjacent risk organs by precisely targeting selected field. In this regard, studies have demonstrated that IMRT with weekly cisplatin reduced gastrointestinal complications for FIGO stage Ib2-Iva cervical cancer patients after radical surgery (38). Therefore, IMRT was used in the current study and the adverse events were not significantly increased even in the extended filed CCRT.

Taken together, the current study demonstrated that efficacy of extended (abdominopelvic) filed-IMRT concurrent with chemotherapy was similar to that of pelvic-IMRT plus chemotherapy; that extended-field IMRT did not increase adverse events of hematology and gastrointestinal tract.

\section{Acknowledgments}

Funding: This study was supported by Natural Science Research Project of Minhang District, Shanghai (grant number: 2015MHZ076).

\section{Footnote}

Reporting Checklist: The authors have completed the CONSORT reporting checklist. Available at http://dx.doi. org/10.21037/tcr-20-2573

Data Sharing Statement: Available at http://dx.doi. org/10.21037/tcr-20-2573

Conflicts of Interest: All authors have completed the ICMJE uniform disclosure form (available at http://dx.doi. org/10.21037/tcr-20-2573). The authors have no conflicts of interest to declare.

Ethical Statement: The authors are accountable for all aspects of the work in ensuring that questions related to the accuracy or integrity of any part of the work are appropriately investigated and resolved. The study was conducted in accordance with the Declaration of Helsinki (as revised in 2013). This study was approved by IRB committee of the Fudan University. The clinical registration number of this study is ChiCTR-IPR-14005499. All procedures performed in studies involving human participants were in accordance with the ethical standards of the institutional and/or national research committee. All patients gave their written informed consent.

Open Access Statement: This is an Open Access article distributed in accordance with the Creative Commons Attribution-NonCommercial-NoDerivs 4.0 International License (CC BY-NC-ND 4.0), which permits the noncommercial replication and distribution of the article with the strict proviso that no changes or edits are made and the original work is properly cited (including links to both the formal publication through the relevant DOI and the license). See: https://creativecommons.org/licenses/by-nc-nd/4.0/.

\section{References}

1. Green JA, Kirwan JM, Tierney JF, et al. Survival and recurrence after concomitant chemotherapy and radiotherapy for cancer of the uterine cervix: a systematic review and meta-analysis. Lancet 2001;358:781-6.

2. ACS. Survival Rates for Cervical Cancer. American Cancer Society. Available online: https://www.cancer.org/cancer/ cervical-cancer/detection-diagnosis-staging/survival.html. Accessed March 102020.

3. Stehman FB, Bundy BN, DiSaia PJ, et al. Carcinoma of the cervix treated with radiation therapy. I. A multivariate analysis of prognostic variables in the Gynecologic Oncology Group. Cancer 1991;67:2776-85.

4. Song S, Kim JY, Kim YJ, et al. The size of the metastatic lymph node is an independent prognostic factor for the patients with cervical cancer treated by definitive radiotherapy. Radiother Oncol 2013;108:168-73.

5. Oh J, Seol KH, Lee HJ, et al. Prophylactic extendedfield irradiation with concurrent chemotherapy for pelvic lymph node-positive cervical cancer. Radiat Oncol J 2017;35:349-58.

6. Sakuragi N, Satoh C, Takeda N, et al. Incidence and distribution pattern of pelvic and paraaortic lymph node metastasis in patients with Stages IB, IIA, and IIB cervical carcinoma treated with radical hysterectomy. Cancer 1999;85:1547-54.

7. Delgado G, Bundy B, Zaino R, et al. Prospective surgicalpathological study of disease-free interval in patients with stage IB squamous cell carcinoma of the cervix: a Gynecologic Oncology Group study. Gynecol Oncol 1990;38:352-7.

8. Samlal RA, van der Velden J, Schilthuis MS, et al. Identification of high-risk groups among node-positive 
patients with stage IB and IIA cervical carcinoma. Gynecol Oncol 1997;64:463-7.

9. Fuller AF, Jr., Elliott N, Kosloff C, et al. Determinants of increased risk for recurrence in patients undergoing radical hysterectomy for stage IB and IIA carcinoma of the cervix. Gynecol Oncol 1989;33:34-9.

10. Wu Y, Zhu B, Han J, et al. A comparative dosimetric study of cervical cancer patients with para-aortic lymph node metastasis treated with volumetric modulated arc therapy vs. 9-field intensity-modulated radiation therapy. Ann Transl Med 2019;7:675.

11. Peters WA 3rd, Liu PY, Barrett RJ 2nd, et al. Concurrent chemotherapy and pelvic radiation therapy compared with pelvic radiation therapy alone as adjuvant therapy after radical surgery in high-risk early-stage cancer of the cervix. J Clin Oncol 2000;18:1606-13.

12. Rotman M, Pajak TF, Choi K, et al. Prophylactic extended-field irradiation of para-aortic lymph nodes in stages IIB and bulky IB and IIA cervical carcinomas. Ten-year treatment results of RTOG 79-20. JAMA 1995;274:387-93.

13. Mabuchi S, Okazawa M, Isohashi F, et al. Postoperative whole pelvic radiotherapy plus concurrent chemotherapy versus extended-field irradiation for early-stage cervical cancer patients with multiple pelvic lymph node metastases. Gynecol Oncol 2011;120:94-100.

14. Zhang G, Fu C, Zhang Y, et al. Extended-field intensitymodulated radiotherapy and concurrent cisplatin-based chemotherapy for postoperative cervical cancer with common iliac or para-aortic lymph node metastases: a retrospective review in a single institution. Int J Gynecol Cancer 2012;22:1220-5.

15. Leath CA, 3rd, Monk BJ. Twenty-first century cervical cancer management: A historical perspective of the gynecologic oncology group/NRG oncology over the past twenty years. Gynecol Oncol 2018;150:391-7.

16. Taylor A, Rockall AG, Reznek RH, et al. Mapping pelvic lymph nodes: guidelines for delineation in intensitymodulated radiotherapy. Int J Radiat Oncol Biol Phys 2005;63:1604-12.

17. Rose PG, Lappas PT. Analysis of the cost effectiveness of concurrent cisplatin-based chemoradiation in cervical cancer: implications from five randomized trials. Gynecol Oncol 2000; 78:3-6.

18. NCI. Common Terminology Criteria for Adverse Events. 2006.

19. Gouy S, Morice P, Narducci F, et al. Nodal-staging surgery for locally advanced cervical cancer in the era of
PET. Lancet Oncol 2012;13:e212-20.

20. McMahon CJ, Rofsky NM, Pedrosa I. Lymphatic metastases from pelvic tumors: anatomic classification, characterization, and staging. Radiology 2010;254:31-46.

21. Whitney CW, Sause W, Bundy BN, et al. Randomized comparison of fluorouracil plus cisplatin versus hydroxyurea as an adjunct to radiation therapy in stage IIB-IVA carcinoma of the cervix with negative paraaortic lymph nodes: a Gynecologic Oncology Group and Southwest Oncology Group study. J Clin Oncol 1999;17:1339-48.

22. Monk BJ, Cha DS, Walker JL, et al. Extent of disease as an indication for pelvic radiation following radical hysterectomy and bilateral pelvic lymph node dissection in the treatment of stage IB and IIA cervical carcinoma. Gynecol Oncol 1994;54:4-9.

23. Matsuo K, Shimada M, Saito T, et al. Risk stratification models for para-aortic lymph node metastasis and recurrence in stage IB-IIB cervical cancer. J Gynecol Oncol 2018;29:e11.

24. Lagasse LD, Creasman WT, Shingleton HM, et al. Results and complications of operative staging in cervical cancer: experience of the Gynecologic Oncology Group. Gynecol Oncol 1980;9:90-8.

25. Tang Y. Analysis on the risk factors of pelvic and paraabdominal aorta lymph node metastasis of endometrial cervical cancer. Shandong University; 2011.

26. Zhou Y. Hysterectomy of early stage cervical cancer with retroperitoneum lymph node metastasis. Fudan University; 2011.

27. Berman ML, Keys H, Creasman W, et al. Survival and patterns of recurrence in cervical cancer metastatic to periaortic lymph nodes (a Gynecologic Oncology Group study). Gynecol Oncol 1984;19:8-16.

28. Eifel PJ, Winter K, Morris M, et al. Pelvic irradiation with concurrent chemotherapy versus pelvic and paraaortic irradiation for high-risk cervical cancer: an update of radiation therapy oncology group trial (RTOG) 90-01. J Clin Oncol 2004;22:872-80.

29. Manders DB, Sims TT, Bailey A, et al. The Significance of Para-Aortic Nodal Size and the Role of Adjuvant Systemic Chemotherapy in Cervical Cancer: An Institutional Experience. Am J Clin Oncol 2018;41:1225-30.

30. Morris M, Eifel PJ, Lu J, et al. Pelvic radiation with concurrent chemotherapy compared with pelvic and paraaortic radiation for high-risk cervical cancer. $\mathrm{N}$ Engl J Med 1999;340:1137-43.

31. Zhang G, He F, Fu C, et al. Definitive extended field 
intensity-modulated radiotherapy and concurrent cisplatin chemosensitization in the treatment of IB2-IIIB cervical cancer. J Gynecol Oncol 2014;25:14-21.

32. Cheng X, Cai SM, Li ZT, et al. Concurrent chemotherapy and adjuvant extended field irradiation after radical surgery for cervical cancer patients with lymph node metastases. Int J Gynecol Cancer 2008;18:779-84.

33. Chen CC, Lin JC, Jan JS, et al. Definitive intensitymodulated radiation therapy with concurrent chemotherapy for patients with locally advanced cervical cancer. Gynecol Oncol 2011;122:9-13.

34. Lv X, Yu H, Chen L. Analysis on the risk factors of paraaorta lymph node metastasis of cervical cancer. Modern Practical Medicine (Chinese) 2011;23:917-18.

35. Liu Y, Zhao LJ, Li MZ, et al. The Number of Positive Pelvic Lymph Nodes and Multiple Groups of Pelvic

Cite this article as: Luo W, Li Y, Ke G, Wu X, Huang X. Extended field or pelvic intensity-modulated radiotherapy with concurrent cisplatin chemotherapy for the treatment of post-surgery multiple pelvic lymph node metastases in cervical cancer patients: a randomized, multi-center phase II clinical trial. Transl Cancer Res 2021;10(1):361-371. doi: 10.21037/tcr20-2573
Lymph Node Metastasis Influence Prognosis in Stage IAIIB Cervical Squamous Cell Carcinoma. Chin Med J (Engl) 2015;128:2084-9.

36. Yap ML, Cuartero J, Yan J, et al. The role of elective paraaortic lymph node irradiation in patients with locally advanced cervical cancer. Clin Oncol (R Coll Radiol) 2014;26:797-803.

37. Asiri MA, Tunio MA, Mohamed R, et al. Is extendedfield concurrent chemoradiation an option for radiologic negative paraaortic lymph node, locally advanced cervical cancer? Cancer Manag Res 2014;6:339-48.

38. Lee J, Lin JB, Chang CL, et al. Prophylactic lower paraaortic irradiation using intensity-modulated radiotherapy mitigates the risk of para-aortic recurrence in locally advanced cervical cancer: A 10-year institutional experience. Gynecol Oncol 2017;146:20-6. 